This item was submitted to Loughborough's Research Repository by the author.

Items in Figshare are protected by copyright, with all rights reserved, unless otherwise indicated.

\title{
Qualitative and quantitative demonstration of bead-to-bead transfer with bone marrow-derived human mesenchymal stem cells on microcarriers: utilising the phenomenon to improve culture performance
}

\section{PLEASE CITE THE PUBLISHED VERSION}

https://doi.org/10.1016/j.bej.2017.11.005

\section{PUBLISHER}

(C) Elsevier

\section{VERSION}

AM (Accepted Manuscript)

\section{PUBLISHER STATEMENT}

This work is made available according to the conditions of the Creative Commons Attribution-NonCommercialNoDerivatives 4.0 International (CC BY-NC-ND 4.0) licence. Full details of this licence are available at: https://creativecommons.org/licenses/by-nc-nd/4.0/

\section{LICENCE}

CC BY-NC-ND 4.0

\section{REPOSITORY RECORD}

Rafiq, Qasim A., Steven Ruck, Mariana P. Hanga, Thomas R.J. Heathman, Karen Coopman, Alvin W. Nienow, David Williams, and Christopher J. Hewitt. 2017. "Qualitative and Quantitative Demonstration of Bead-to-bead Transfer with Bone Marrow-derived Human Mesenchymal Stem Cells on Microcarriers: Utilising the Phenomenon to Improve Culture Performance". figshare. https://hdl.handle.net/2134/27820. 


\section{Qualitative and quantitative demonstration of bead-to-bead transfer with bone marrow-derived human mesenchymal stem cells on microcarriers: utilising the phenomenon to improve culture performance}

Qasim A. Rafiq1,2,3, Steven Ruck ${ }^{3}$, Mariana P. Hanga2,3, Thomas R.J. Heathman ${ }^{3,4}$, Karen Coopman ${ }^{3}$, Alvin W. Nienow2,3,5 David J. Williams ${ }^{3}$ and Christopher J. Hewitt2,3

${ }^{1}$ Advanced Centre for Biochemical Engineering, Department of Biochemical Engineering, University College London, Gower Street, London, WC1E 6BT, United Kingdom

${ }^{2}$ Aston Medical Research Institute, School of Life and Health Sciences, Aston University, Aston Triangle, Birmingham, B4 7ET, United Kingdom

${ }^{3}$ Centre for Biological Engineering, Loughborough University, Leicestershire, LE11 3TU, United Kingdom

4PCT, a Hitachi Group Company, 4 Pearl Ct, Allendale, New Jersey 07401, USA

${ }^{5}$ School of Chemical Engineering, University of Birmingham, Edgbaston, Birmingham, B15 2TT, United Kingdom

Correspondence: Dr Qasim Rafiq, Advanced Centre for Biochemical Engineering, Department of Biochemical Engineering, University College London, Gower Street, London, WC1E 6BT, United Kingdom

Keywords: human mesenchymal stem cells; cell therapy bioprocessing; microcarriers; bioreactor; regenerative medicine; bead to bead transfer; process intensification 


\section{Abstract}

Formatted: Font color:

Human mesenchymal stem cells (hMSCs) are a key candidate for advanced cell therapies with numerous clinical trials investigating their potential to treat acute and chronic indications. However, important translational and manufacturing challenges need to be addressed to improve our capability for scalable production of fully functional cells. In this study, we have demonstrated, both qualitatively and quantitatively, the ability of bone marrow-derived hMSCs to migrate from one microcarrier to another, and, to populate fresh microcarriers when added into suspension culture. Additionally, we have shown that compared to inoculating a culture with cells in free suspension, inoculating $10 \%$ of near-confluent microcarriers from an initial seed microcarrier culture resulted in an increase in the cell growth rate and overall cell yield and a significant reduction in the lag phase. These findings were consistent across cells from three different BMhMSC donors and across different culture medium conditions, foetal bovine serum-supplemented medium, human platelet lysate-supplemented medium and serum-free medium. This new cellson-beads inoculation method is an effective means of process intensification with the potential to decrease manufacturing times and potentially costs of hMSC-based therapies. 


\section{Introduction}

Advanced therapies, including cell and gene-based therapies, will form the next generation of therapeutics which have the potential to address unmet clinical need, treat chronic conditions and offer potential cures rather than simply alleviating patient symptoms (Barry and Murphy 2013). Adherent, or anchorage-dependent cells, are likely to be promising candidates for both autologous (patient-specific) and allogeneic (universal donor) cell-based therapies; such cells include human adult mesenchymal stem cells (hMSCs), human embryonic stem cells (hESCs) and human induced pluripotent stem cells (hiPSCs). However, the growth of adherent cells presents additional challenges in comparison to suspension cell platforms, and has therefore been the subject of considerable research activity (Rafiq 2016). Initially in the 1980s and 1990s, significant effort was directed at the production of adherent Chinese Hamster Ovary (CHO) cells for protein/vaccine production, and more recently, for the production of adherent stem cells for cell therapy applications. Established techniques for the growth of these cells involve monolayer culture, predominantly in tissue-culture flasks or roller bottles. However, as was recognised for adherent $\mathrm{CHO}$ cells in the 1980s, alternative manufacturing processes for the production of adherent cells are required which are scalable, robust, reproducible and cost-effective. This is achieved through the use of microcarriers in stirred-tank bioreactor systems and have been used for multiple cell types including CHO (Ohlson et al. 1994), hMSCs (Dos Santos et al. 2011; Rafiq et al. 2013a), hESCs (Chen et al. 2010) and hiPSCs (Badenes et al. 2017; Bardy et al. 2013).

In monolayer culture, cell growth is maintained by keeping the cells in the log phase of the culture and is based upon 'confluency', the proportion of adherent cells covering the attachment surface.

\begin{tabular}{|l|}
\hline Field Code Changed \\
\hline Field Code Changed \\
\hline Field Code Changed \\
\hline Field Code Changed \\
\hline Field Code Changed \\
\hline Field Code Changed \\
\hline
\end{tabular}
When cells reach an appropriate confluency level (usually 80\% but can differ based on cell type), the cells are removed from the surface and plated into new tissue culture flasks in order to keep 
them in the exponential phase, a process known as passaging. The process of passaging typically requires the use of a proteolytic enzyme such as trypsin, dispase or collagenase (Lindner et al. 1987) in conjunction with a chelating agent such as EDTA. The exposure of adherent cells to these reagents in the absence of serum-containing medium enables the digestion and cleaving of cell attachment proteins. However, frequent exposure to such reagents has been demonstrated to have adverse effects on mammalian cells including proteome alteration (Huang et al. 2010) and effects on cell shape and chromatin structure (Kapiszewska et al. 1991). As such there is concern regarding the consecutive passaging of cells, in part due to the long-term deleterious effects of dissociation reagents on the cell cytoskeleton (Madeira et al. 2012). Although enzyme-free dissociation buffers also exist, some formulations may not be suitable for use with hMSCs. For example, a study using a Gibco-brand enzyme-free dissociation buffer to detach hMSCs grown on tissue culture plastic found that not only was cell viability following detachment significantly reduced compared to trypsin controls ( 70\% compared to > $90 \%$, respectively) but cells also failed to reattach to tissue culture plastic (Heng et al. 2009).

Until now, hMSC microcarrier culture has followed the same approach as monolayer culture with respect to harvesting when cells reach confluency, i.e. the use of enzymes to detach cells from the surface of the microcarriers, preferably aided by agitation to reduce the time the cells are exposed to that environment (Nienow et al. 2014). However, unlike monolayer culture which is limited by the surface area available in the tissue culture flask, microcarrier/bioreactor culture has a significant advantage with respect to surface area to volume ratio and there is potential for the provision of additional microcarriers during a culture process when the cells are nearing confluency in order to maintain cell growth in the exponential phase for longer. For example, Hervy and colleagues recently maintained hMSCs on microcarriers for over 40 days using this 
approach (Hervy et al. 2014). This extended culture time relies on the transfer of cells from one microcarrier/bead to another (called here for convenience, bead-to-bead transfer). Bead-to-bead transfer (or cell migration from one microcarrier to another) during suspension culture of adherent cells is believed to be a key mechanism for rapid and efficient cell expansion in suspension cultures (de Soure et al. 2016). This cell behaviour is of particular interest from a process development and manufacturing perspective as the demonstration of bead-to-bead transfer would facilitate the large-scale manufacture of stem cells via seed bioreactor cultures without the need to expose the cells to frequent deleterious proteolytic enzymes. This approach also facilitates process intensification by providing additional surface throughout the course of the culture, maintaining the cells in the exponential phase of growth for a longer period of time, thereby increasing yield and efficiency whilst reducing process costs and time.

Although investigated and proven for adherent $\mathrm{CHO}$ and Vero cells (Ohlson et al. 1994; Wang and Ouyang 1999), work demonstrating such a phenomenon for hMSCs has to date focused on single hMSC donors (Takahashi et al. 2017), or an immortalised cell line (hMSC-TERT) (Leber et al. 2017) or it included complex agitation regimes (Hervy et al. 2014). In this study, we demonstrate, both qualitatively and quantitatively, the phenomenon of bead-to-bead transfer for three donor BM-hMSCs on different microcarriers (Plastic P102-L, Cytodex-3 and Hillex) in different culture medium conditions, foetal bovine serum (FBS), human platelet lysate (HPL) and serum-free medium (SFM). We also explore the use of a cells-on-beads inoculation procedure, which subsequently utilises bead-to-bead transfer (henceforth called 'cells-on-beads inoculation'), in contrast to the standard free suspension inoculation procedure to facilitate process intensification. Several important parameters were measured throughout the culture including cell

\section{Field Code Changed}

Field Code Changed

Field Code Changed

Field Code Changed

Field Code Changed

Field Code Changed

Field Code Changed 
growth kinetics, viability, key metabolite consumption and production, immune-phenotype and differentiation potential.

\section{Materials and methods}

\section{1 hMSC monolayer expansion}

Human MSCs from three donors were used in this study. All were isolated from bone-marrow aspirate and obtained from Lonza (Walkersville, USA) after the donors provided informed consent. The university's local Ethical Committee approved the use of the samples for research. The isolation and culture of primary hMSCs is described in Rafiq et al. (2013b); in brief, hMSCs were isolated on the basis of plastic adherence and cryopreserved at passage 1 at a density of 2 x $10^{6}$ cells $/ \mathrm{mL}$ in $10 \%$ dimethyl sulphoxide (DMSO) (v/v) (Sigma Aldrich, UK) and $90 \%$ foetal bovine serum (FBS; Hyclone, Lot\# RUF35869). The cells were cultured according to previously determined protocols (Rafiq et al., 2013; Heathman et. al., 2015). Briefly, the cells were seeded at 5,000 cells $/ \mathrm{cm}^{2}$ and grown on tissue culture plastic in DMEM (1 g/L glucose, Lonza, UK) supplemented with $10 \%(\mathrm{~V} / \mathrm{v})$ foetal bovine serum (FBS; HyClone) or 10\% (v/v) non-heparin requiring (PL-NH) Stemulate ${ }^{\mathrm{TM}}$ (Cook Regentec, USA) for the human platelet lysate based culture conditions, and $2 \mathrm{mM}$ UltraGlutamine (Lonza, UK).

Where cells were also cultured under serum-free medium (SFM) conditions, the Prime-XVTM SFM hMSC medium was used (Irvine Scientific, USA) in accordance with the manufacturer's instructions. As required, attachment surfaces were pre-coated with recombinant fibronectin (Irvine Scientific, USA). For both the SFM and HPL cultures, the BM-hMSCs underwent one adaptation passage in medium containing either the Prime-XVTM medium or 10\% (v/v) PL-NH Stemulate ${ }^{\mathrm{TM}}$. 


\subsection{Spinner flask microcarrier culture}

The spinner flask cultures involved the use of $100 \mathrm{~mL}$ BellCo spinner flasks (BellCo, USA), with a $100 \mathrm{~mL}$ working volume and a vessel diameter $(\mathrm{T})$ of $60 \mathrm{~mm}$. The spinner flask vessels were siliconized prior to use with Sigmacote (Sigma Aldrich, UK) as described in (Rafiq et al. 2016). The spinner flasks were agitated by a magnetic stirrer bar at the just suspended speed ( $\mathrm{N}_{\mathrm{s}}$ ) placed on the Bell-Ennium ${ }^{\mathrm{TM}}$ Compact 5 position magnetic stirrer platform (BellCo, USA) with air in the headspace for surface aeration giving essentially $100 \% \mathrm{dO}_{2}$. The microcarriers were prepared according to the manufacturer's instructions and sterilised by autoclaving at $121^{\circ} \mathrm{C}$ and $1 \mathrm{~atm}$ for 15 minutes. Prior to use, the microcarriers were conditioned in growth medium for at least 1 hour to facilitate cell attachment. The surface area provided per spinner flask was $500 \mathrm{~cm}^{2}$ and the cells were inoculated at a density of 6,000 cells $/ \mathrm{cm}^{2}$ as previously described (Rafiq et. al., 2013; Heathman et. al., 2015). This inoculation method, referred to later as the 'control', is in contrast to the 'cells-on-beads' inoculation method (discussed later in the results section) which involved inoculating $10 \%$ of near-confluent microcarriers from an initial seed microcarrier culture. In all cases, a 50\% medium exchange was performed at $72 \mathrm{~h}$ in culture and then every $48 \mathrm{~h}$ thereafter.

\subsection{Analytical Techniques}

Cell counts and viability (via acridine orange uptake and DAPI exclusion) were determined using the Nucleocounter NC-3000 (Chemometec, UK). Cell viability was also assessed by the Live/Dead Viability/Cytotoxicity kit (Calcein AM/ Ethidium Homodimer; Life Sciences, ThermoFisher, UK) following the manufacturer's instructions. Briefly, the cells were first washed with PBS and then incubated with the Live/Dead stain containing a final concentration of $2 \mu \mathrm{M}$ 
calcein-AM and $4 \mu \mathrm{M}$ ethidium homodimer in the dark at $37^{\circ} \mathrm{C}$ and $5 \% \mathrm{CO}_{2}$ for 40 minutes. Post incubation, the Live/Dead working solution was removed and replaced with PBS. Samples were then visualised on a Nikon Ti Eclipse epi-fluorescence microscope (Nikon, UK).

Analysis of glucose and lactate concentrations in the spent medium was performed using a Cedex Bio-HT (Roche, Germany). The following parameters were calculated from the data obtained:

1. Specific Growth Rate

$$
\text { Specific growth rate, } \mu=\frac{\ln \left(c_{x}(t) / c_{x}(0)\right.}{\Delta t}
$$

where $\mu$ is the net specific growth rate $\left(h^{-1}\right), C_{x}(t)$ and $C_{x}(0)$ are the cell numbers at the end and start of the exponential growth phase, respectively and $t$ is time $(\mathrm{h})$.

2. Population Doublings

$$
\text { Population Doublings, } P_{d}=\frac{1}{\log (2)} \cdot \log \left(\frac{C_{x}(t)}{C_{x}(0)}\right)
$$

where $P_{d}$ is the number of population doublings, $C_{x}(t)$ and $C_{x}(0)$ are the cell numbers at the end and start of the exponential growth phase, respectively.

3. Specific Metabolite Consumption/Production Rate

$$
\text { Specific metabolite flux, } q_{m e t}=\left(\frac{\mu}{C_{x}(0)}\right) \cdot\left(\frac{C_{m e t}(t)-C_{m e t}(0)}{e^{\mu t}-1}\right)
$$

where $q_{\text {met }}$ is the net specific metabolite consumption or production rate, $\mu$ is the specific growth rate $\left(h^{-1}\right), C_{x}(0)$ is the cell number at the end of the exponential growth phase, $C_{\text {met }}(t)$ and $C_{\text {met }}(0)$ 
are the metabolite concentrations at the end and start of the exponential growth phase, respectively and $t$ is time (h).

\section{4 hMSC Characterisation}

Immunophenotype analysis was performed by multiparameter flow cytometry before and after the hMSC expansion process using a previously developed protocol (Chan et al. 2014). In brief, this involved the use of flow cytometry where harvested, single cells were suspended in growth medium and loaded onto a 96 well plate. The cells were stained with monoclonal antibodies against the positive hMSC markers, CD73, CD90 and CD105 and the negative markers, CD34 \& HLA-DR (BD Biosciences, UK) in addition with the corresponding isotype controls (BD Biosciences, UK). The antibodies selected were those recommended by the International Society for Cellular Therapy (ISCT) for the characterisation of hMSCs (Dominici et al. 2006).

Field Code Changed

The hMSC differentiation was induced using Prime-XVTM Differentiation SFM (Irvine Scientific, USA) as per manufacturer's instructions. After 21 days, the differentiation media were removed, cells rinsed with PBS then fixed with 4\% (v/v) PFA at room temperature. Adipocytes were stained with 1\% (w/v) Oil Red O (Sigma-Aldrich, UK) in isopropanol at room temperature and rinsed with distilled water. Osteoblasts were incubated with 2.5\% (v/v) silver nitrate (Sigma-Aldrich, UK) under ultraviolet light (30 minutes exposure), rinsed with distilled water and stained with fast violet solution (Sigma-Aldrich, UK) containing 4\% (v/v) napthol AS-MX phosphate alkaline (SigmaAldrich, UK) for 45 minutes at room temperature in the dark. Chondrocytes were stained with $1 \%$ (w/v) Alcian blue (Sigma-Aldrich, UK) in $0.1 \mathrm{M}$ hydrochloric acid (Sigma-Aldrich, UK). After 30 minutes incubation, cells were rinsed three times with $0.1 \mathrm{M} \mathrm{HCl}$. After staining, differentiated cells were visualized under a light microscope (Nikon Eclipse TS-100, UK). 


\subsection{Tracking of bead-to-bead cell transfer:}

Bead-to-bead cell transfer was assessed by two methods: 1) by employing visually distinctive microcarriers in the same culture system (adding a visually distinctive microcarrier at a later stage of the culture) and 2) by fluorescently labelling cells with two different Cell TrackerTM dyes (Life Technologies, ThermoFisher Scientific, UK). The use of visually distinctive microcarriers provides an effective way of demonstrating bead-to-bead transfer. The cells were originally cultured on the Plastic P102-L microcarriers in agitated spinner flask conditions (as described above). After 3 days of culture, the visually distinctive microcarriers (either the Hillex or Cytodex-1) were added at the same microcarrier concentration during a 50\% medium exchange. The culture was continuously agitated throughout the addition of the second microcarrier type so that the microcarriers were never static. This procedure unequivocally demonstrated the transfer of cells from the Plastic P102-L microcarrier to the new microcarrier (either Hillex or Cytodex-1).

For the fluorescent labelling, Cell Tracker ${ }^{\mathrm{TM}}$ Green CMFDA and Cell Tracker ${ }^{\mathrm{TM}}$ Red CM-Dil (Life Technologies, ThermoFisher Scientific, UK) were employed. Cell Tracker ${ }^{\mathrm{TM}}$ Green contains chloromethyl derivatives of fluorescein diacetate (CMFDA) and is nonfluorescent until cytosolic esterases cleave off the acetates, releasing a brightly fluorescent product. Unlike the Cell Tracker $^{\mathrm{TM}}$ Green CMFDA, Cell Tracker ${ }^{\mathrm{TM}}$ Red CM-Dil is a lipophilic carbocyanine that incorporates a mildly thiol-reactive chloro-methyl substituent with affinity for the lipids present

Formatted: Font color: Auto

Formatted: Font color: Auto 
inside the cell membrane. These reagents pass freely through cell membranes, but once inside the cell, are transformed into cell-impermeant reaction products.

The Cell Tracker ${ }^{\mathrm{TM}}$ dyes were used according to the manufacturer's instructions. Briefly, based on the average cell concentration obtained post-harvest, the volume of cell suspension required for each experiment to achieve a cell density of $10,000 \mathrm{cell} / \mathrm{s} / \mathrm{cm}^{2}$ was calculated and split in equal volumes in two separate centrifuge tubes. Cell pellets were then obtained by centrifuging the cell suspensions at $200 \mathrm{~g}$ for 5 minutes. Each cell pellet was then resuspended in a working solution of Cell Tracker ${ }^{\mathrm{TM}}$ Red CM-Dil (working concentration of $20 \mu \mathrm{M}$ ) and Cell Tracker ${ }^{\mathrm{TM}}$ Green CMFDA (working concentration of $20 \mu \mathrm{M}$ ), respectively, and incubated in the dark at $37^{\circ} \mathrm{C}$ for 40 minutes. After incubation, the Cell Tracker ${ }^{\mathrm{TM}}$ working solutions were removed by centrifugation and replaced with fresh pre-warmed growth medium. The fluorescently-labelled cells were then seeded at 10,000 cells $/ \mathrm{cm}^{2}$ on the pre-conditioned microcarriers in spinner flasks. Combinations of two different microcarriers (Plastic and Cytodex-1) and two different fluorescent dyes were used for this study. The labelled cells were allowed to attach and proliferate for $48 \mathrm{~h}$ in agitated conditions, in the dark, at $37^{\circ} \mathrm{C}$ and $5 \% \mathrm{CO}_{2}$. After $48 \mathrm{~h}$ in culture, the different microcarrierlabelled cell suspensions were mixed in a 50:50 proportion and the culture was continued for an additional 2 days. The mixed microcarrier-labelled cell suspensions were then imaged on the epifluorescence microscope mentioned above.

\subsection{Statistical analysis}

For comparison between the two data sets comparing the mean specific growth rates, statistical significance was determined by using the Student's two-tailed t-test. Significance was determined

Formatted: Font color: Auto 
at $p<0.05\left(^{(}\right)$or $p<0.01\left(^{(\star)}\right)$ GraphPad Prism 6 (California, USA) was used for the statistical analysis 


\section{Results and Discussion}

The aim of this work was to demonstrate, both quantitatively and qualitatively, the phenomenon of bead-to-bead transfer of hMSCs on microcarriers under different culture conditions including cells from three BM-hMSC donors and under different culture medium conditions, FBS, HPL and SFM. An additional aim was to investigate the potential for process intensification through cells-onbeads inoculation, where $10 \%$ of near-confluent microcarriers were used to inoculate a fresh microcarrier culture.

Cell-microcarrier contact in bead-to-bead hMSC transfer is very likely to be influenced by a combination of factors such as donor cell line, medium composition, attachment substrate (i.e. microcarriers) and the process itself. The process employed with respect to the feeding regime, surface area provided or agitation intensity was kept constant during this study using a protocol that had been thoroughly tested and optimised for the successful expansion of bone-marrow derived hMSCs in spinner flasks in different medium conditions (Heathman et al. 2015; Rafiq et al. 2016). To account for donor variability, hMSCs from three donors were employed for this

Field Code Changed

Field Code Changed study.

\subsection{Qualitative demonstration of bead-to-bead transfer}

To qualitatively demonstrate hMSC migration from one microcarrier to another, two different methods were employed. The first method made use of commercially available microcarriers with different visual properties, while the second method consisted of fluorescently-labelling cells, followed by tracking their movements in culture.
Formatted: Font color: 
Prior work with multiple types of microcarriers in a microcarrier screening study (Rafiq et al. 2016) showed that BM-hMSCs would attach and grow on most of the microcarriers albeit with varying degrees of success on each. It was also found that certain microcarriers were visually distinctive compared to others, for example the Cytodex-1 microcarriers are translucent in comparison to the solid Plastic P102-L microcarriers, and the Hillex microcarriers absorb the phenol red present in the medium and therefore turn a dark red colour. To demonstrate the phenomenon of hMSC bead-to-bead transfer qualitatively, the BM-hMSCs were initially cultured exclusively on Plastic P102-L microcarriers for a period of 6 days, after which fresh Hillex microcarriers (Figure 1A-C) or fresh Cytodex-1 microcarriers (Figure 1D-F) were aseptically added to the culture without stopping agitation of the culture at any point.

As is indicated by the arrows in the figures, it is evident that BM-MSCs were attached to both the Plastic P102-L microcarriers (solid white) and the Hillex (dark red) microcarriers (Figure 1A-C). Similarly, cells were attached to the Plastic P102-L and Cytodex 1 (translucent white) microcarriers (Figure 1D-F). The BM-hMSCs were found to be growing on microcarriers of both types as either cell-microcarriers aggregates, as well as on individual Plastic P102-L, Hillex and Cytodex-1 microcarriers (Figure 1A-F). This observation is consistent with both the bridging and cell migration methods of bead-to-bead transfer noted by Leber and colleagues in their studies using an immortalised hMSC line (Leber et al. 2017). It is worth noting that following the addition of the second type of microcarrier to the culture vessel, at no point was the agitation stopped and $\mathrm{N}_{\mathrm{JS}}$ was maintained throughout. These findings therefore provide a qualitative demonstration of bead-to-bead transfer during a continuing culture, whereby hMSCs have attached and proliferated on 'fresh' microcarriers days after the addition to the culture.

Field Code Changed 
To demonstrate that these were indeed viable cells attached to both types of microcarriers (Plastic P102-L and Hillex), samples were stained with calcein AM (viable stain, green) and ethidium homodimer (non-viable stain, red) and assessed under a fluorescent microscope (Figure 2). It is evident that the cells which are attached to the microcarriers in phase contrast images are indeed viable cells given the extent of viability staining exhibited in Figures 2B and 2D. Similar findings were found with the Plastic P102-L and Cytodex-1 pairing and the results depicted in Figure 2 are representative of that combination of microcarriers too.

To further demonstrate the bead-to-bead transfer qualitatively, the cells were fluorescently labelled with two different fluorescent dyes prior to inoculation on the microcarriers. These Cell Tracker $^{\mathrm{TM}}$ dyes are virtually non-fluorescent until cytosolic esterases found only inside living cells cleave off the acetate moiety, releasing a brightly fluorescent product. Moreover, these reagents pass freely through cell membranes, but once inside the cell, are transformed into cellimpermeant reaction products. Further, the fluorescent probes are inherited by the daughter cells and are not transferrable to adjacent cells in a population. As such, the Cell Tracker ${ }^{\mathrm{TM}}$ Redlabelled cells were cultured initially only onto Plastic microcarriers (solid) and the Cell Tracker $^{\mathrm{TM}}$ Green-labelled cells cultured initially only onto the Cytodex-1 microcarriers (transparent) for 2 days. After combining the two different types of microcarriers, there was evidence that Cell Tracker $^{\mathrm{TM}}$ Red-labelled cells have migrated from Plastic onto Cytodex-1 microcarriers (white arrows) and vice versa (yellow arrows) (Figure 3A-3D). Notably, the migrated cells exhibited a more rounded morphology than these adherent cells normally do. During mitosis, adherent cells undergo a cycle of detachment and re-attachment (Yamakita et al. 1999) so this could be indicating active cell growth on the 'new' microcarrier that the cell has migrated to or it could be
Formatted: Font color:

Auto

Formatted: Font color:

Field Code Changed 
an indication that following detachment from another microcarrier during mitosis, the cell had not yet fully adhered at the time of imaging.

\subsection{Quantitative demonstration of bead-to-bead transfer}

To quantitatively demonstrate bead-to-bead transfer, BM-hMSC1 and BM-hMSC2 were grown for 6 days on Plastic P102-L microcarriers in either FBS-based or SFM culture conditions (Figure 4, Culture 1), after which, $10 \%$ of the microcarriers (with cells attached) were seeded into a fresh culture vessel with 90\% fresh Plastic P102-L microcarriers (Figure 4, Culture 2). Upon inoculation of the microcarriers with cells attached, the vessel was immediately agitated at Nss. This was repeated for a third time (Figure 4, Culture 3).

With each consecutive culture, it is noteworthy that the initial lag phase is reduced and with each culture, the final cell density obtained by the end is greater than the previous. For FBS cultures, the final cell density was found to be $\sim 1.6 \times 10^{5} \pm 0.15$ cells $/ \mathrm{mL}$ obtained with Culture $1,2.01 \times$ $10^{5} \pm 0.06$ cells $/ \mathrm{mL}$ in Culture 2 and $\sim 2.15 \times 10^{5} \pm 0.08$ cells $/ \mathrm{mL}$ obtained in Culture 3 for hBMMSC1 and a similar trend was found for hBM-MSC2 (Figure 4). For SFM cultures, the cell yield was higher in all cases, but the same trend of reduced lag phase and increasing yield within the same time period (6 days) was noted. Moreover, in all cases, viability was $>95 \%$, indicating favourable culture conditions in the vessel for the growth of hMSCs throughout the course of each culture. These findings indicate that a $10 \%$ concentration of near-to-confluent microcarriers was not only sufficient to inoculate a new vessel containing $90 \%$ fresh microcarriers, but decreased the lag phase of the new culture and increased the overall yield. 
The findings would appear to demonstrate bead-to-bead transfer given the fact that a $10 \%$ microcarrier concentration (with cells attached) was able to successfully inoculate a culture vessel with fresh microcarriers, with the culture eventually reaching a point of near-confluency prior to termination of the culture. Similar findings were observed by Ohlson et al. (1994) and Wang and Ouyang (1999) for adherent $\mathrm{CHO}$ cells who performed similar inoculation studies with nearconfluent and fresh microcarriers. Moreover, Ohlson et al. (1994) also observed an increase in the overall cell yield and a reduced lag phase, similar to what was found in this study. In addition to investigating a $10 \%$ addition of near-confluent microcarriers to a culture containing fresh microcarriers, Ohlson et al. (1994) also investigated inoculating a 25\% concentration of nearconfluent microcarriers to a fresh culture and found that this resulted in a further reduced lag phase and an even greater cell density than the 10\% near-confluent microcarrier addition. Although a 25\% concentration of near-confluent microcarriers were not investigated in our study, that earlier finding indicates that an important process development activity might be to establish the optimal concentration of near-confluent microcarriers to inoculate into fresh bioreactor systems. This analysis will undoubtedly be a trade-off between the number of bioreactors to be seeded and the desired culture growth kinetics (similar to splitting a near-confluent T-flasks into multiple T-flasks). In a separate study, Badenes et al. (2017), demonstrated bead-to-bead transfer with hIPSCs, where it was found that this technique facilitated an increase in viable cell number and a concomitant significant increase in fold expansion within a 15 day culture.

To further quantitatively demonstrate the phenomenon of bead-to-bead transfer, a fluorescence microscopy-based manual cell counting technique was adopted to highlight the difference between two inoculation methods. One (the control) used the well-established technique of cells in free suspension and the other, used inoculation of an equivalent number of cells attached to 
$10 \%$ of the beads. With these two methods, one would expect there to be a distinct difference in how the distribution of the hMSC population across the microcarriers changed over the culture period. For the control method using cells in suspension, the expectation would be very few cells per microcarrier at the start of the culture and with time as the hMSCs proliferated, this cell distribution would shift to higher numbers of cells per microcarriers. With the inoculation via the bead-to-bead transfer method, the expectation would be that the cell distribution splits into two distinct populations at the start of the culture, one population with a lot of cells, being the microcarriers added with cells attached, and the other being empty microcarriers. As the hMSCs proliferate and bead-to-bead transfer occurs, one would expect these two populations to merge over the course of the growth phase, finally producing similar distributions at the end of the cultures.

The data presented (Figure 5A) reflects the expected trends, particularly in the case of inoculating cells in suspension as is traditionally done (the control) where the number of cells per bead began with many beads without cells at day zero with $75 \%$ of beads with 1 to 5 cells on day 1 . The maximum number of cells per bead steadily increased until eventually at day 5 , the maximum number of beads were those approaching or at confluence with between 5 to 15 cells per bead on the remainder.

With the inoculation via cells on beads followed by bead-to-bead transfer (Figure 5B), few beads have many cells (some approaching confluence) and the remainder have none on day 0 . With the passage of time up to day 5 , fewer and fewer beads are without cells and the number of cells 
is approximately the same on the other beads and steadily increases. At the end (day 5), a very high proportion of the beads are approaching or have reached confluence.

\subsection{Comparison of inoculation techniques}

Having established that BM-hMSCs can transfer to freshly added microcarriers and that a 10\% concentration of near-confluent microcarriers could act as a sufficient inoculum for a fresh culture, to further prove the efficacy of the new approach, it was decided to include cells from a third donor (BM-hMSC 3) to supplement the two used in the experiments establishing bead-to-bead transfer (BM-hMSC 1, BM-hMSC 2). Also a third culture medium (HPL-based) was included. The results inoculating with these three donor cells on Plastic P102-L microcarriers and three different medium are summarised in Figure 6 (FBS-based), Figure 7 (SFM) and Figure 8 (HPL-based). The results are also compared to our previous approach of free suspension inoculation as control (Rafiq et al. (2016); Heathman et al. (2015)). Though the results differed between the different medium, the data were very similar for each donor cell. Thus, the data presented in Figures 6-9 illustrate a typical result for any of the cells in that specific medium conditions.

There was a significant improvement in performance between the two approaches in all culture medium conditions (FBS, HPL and SFM) throughout the course of the culture, resulting in a higher cell density at the end of the culture when utilising cells-on-beads inoculation. For the FBSbased culture, the cell density was $\sim 2.0 \times 10^{5}$ cells $/ \mathrm{mL}$ compared to $\sim 1.5 \times 10^{5}$ cells $/ \mathrm{mL}$ respectively (Figure $6 \mathrm{~A}$ ) and for SFM, the final cell densities were $\sim 4.6 \times 10^{5} \mathrm{cell} / \mathrm{s} / \mathrm{mL}$, compared to $\sim 3.4 \times 10^{5}$ cells $/ \mathrm{mL}$ respectively (Figure $7 \mathrm{~A}$ ). A similar trend was found for HPL, where the values were $\sim 3.6 \times 10^{5}$ and $\sim 3.0 \times 10^{5}$ cells $/ \mathrm{mL}$ respectively (Figure $8 \mathrm{~A}$ ). This increase in cell

Formatted: Font color: Auto

Formatted: Font color: Auto

Formatted: Font color:

Formatted: Font color:

Field Code Changed

Field Code Changed

Formatted: Font color: 
yield is associated with a reduced lag phase for all cultures using the cells-on-beads inoculation method, enabling the cells to reach the higher growth phase of the culture quicker in comparison to the control. This more rapid achievement of fast growth is illustrated in Figures $6-8 \mathrm{~B}$ and Figures 6-8C. Figures 6-8 B, shows that for FBS, SFM and HPL cultures, the cells' mean specific growth rate was significantly higher between days 0-2 with the cells-on-beads inoculation when compared to the controls (Figures 6-8C). After that, the growth rate for each method reached parity between days 2-4. These figures also make clear that there was a reduced lag phase with cells-on-beads inoculation.

The specific glucose consumption rate along with the specific lactate and ammonia production rates were also calculated for each culture (Figure 9). For FBS-based culture, the specific glucose consumption rate during pre-culture for both the inoculation via cells-on-beads and the control method was $\sim 19$ pmol.cell-1. $d^{-1}$. Then between days 0-2 post inoculation, these values sharply rose to $\sim 38$ pmol.cell-1 ${ }^{-1} \mathrm{~d}^{-1}$ for the inoculation via cells-on-beads and was even higher ( $\sim 45$ pmol.cell-1. $\left.\mathrm{d}^{-1}\right)$ for the control. Between days 2-4, the glucose consumption rates have settled back down to similar values of $\sim 20$ pmol.cell-1. $\mathrm{d}^{-1}$ for both methods. This increase in specific glucose consumption rate during the first 2 days of culture was mirrored by an increase in the specific lactate and ammonia production rates during the same time phase. A distinct trend could Formatted: Font color: be seen across each medium type, where in general an increase in specific consumption/production rates over the first 2 days of culture was significantly reduced when using the inoculation via cells-on-beads instead of the control. It must be noted that all rates were significantly reduced when using a serum-free medium. 
This trend suggested that using inoculation by cells-on-beads resulted in a reduced impact on the metabolic activity of the hMSCs during the early phase of culture that can likely be attributed to the production of extracellular components required for attachment when using the control method. Although it has not been explored here, this highlights a potential impact of multiple trypsinisation steps on the potency or long-term proliferative capability of hMSCs which could be avoided by adopting the inoculation via cells on beads followed by bead to bead transfer.

With respect to characterisation, the BM-hMSCs, both pre-inoculation and post-harvest were characterised with respect to cell identity (immunophenotypic expression) and functionality (differentiation potential). In all cases (different culture medium conditions and inoculation methods), the BM-hMSCs were found to be in accordance with the ISCT criteria (Dominici et al. 2006). Representative data is shown for the condition wherein the BM-hMSCs were cultured in SFM and inoculated using cells-on-beads. However similar findings were found for all other conditions (data not shown). The data demonstrates that there was $>95 \%$ co-expression of CD105, CD90 and CD73 and less than 2\% positive for the negative markers CD34 and HLA-DR for the harvested BM-hMSCs (Supplementary Figure 1). Moreover, the cells were analysed for the retention of their tri-lineage differentiation potential towards the osteogenic, chondrogenic and adipogenic pathways. Again, in all cases, the BM-hMSCs were found to retain their differentiation potential (Supplementary Figure 1).

The impact of this study is particularly relevant to the production phase for hMSC manufacture, where the need for large numbers of cells within a limited time period may be necessary. This approach allows for multiple bioreactors to be inoculated and operated in parallel from a small percentage of near-confluent microcarriers, which lends itself well to an allogeneic cell therapy.

Formatted: Font color: Auto 
This production approach is akin to T-flask based culture, where a near-confluent T-flask is 'split' into other T-flasks to facilitate the progression of culture. The new approach offers a significant advantage in that the scale of a stirred-tank bioreactor is significantly larger than that of a T-flask and therefore so is the yield of cells that can be obtained. Combined with the ability to increase surface area during the course of the culture through the addition of fresh microcarriers, this approach facilitates significant expansion potential. Unlike a traditional seed train process from primary cells, this process could enable cells to be cultured at relatively low population doublings in multiple reactors before either being frozen down as a part of a master/working cell bank, or indeed, for the final manufacture of cells. 


\section{Conclusions}

Bead-to-bead transfer is widely believed to occur in anchorage-dependent microcarrier cultures. Unlike previous studies investigating this phenomenon, this study describes the approach taken to demonstrate bead-to-bead transfer, both qualitatively and quantitatively, for three different BMhMSC donors and under different culture medium conditions, FBS, HPL and SFM. Qualitative demonstration of the phenomenon was achieved by using microcarriers with distinctive visual properties and cell-tracking fluorescent reagents where it was demonstrated that freshly added microcarriers to an existing culture were soon populated by BM-hMSCs, with examples of cells bridging between different microcarriers, and in other instances cells attaching and proliferating on the freshly added microcarriers in isolation. Quantitative demonstration of the phenomenon was established by the process of taking $10 \%$ of near-confluent microcarriers to inoculate a fresh microcarrier culture (cells-on-beads inoculation), where it was found that bead-to-bead transfer not only occurred, but that this inoculation method, in contrast to the control (where cells were inoculated via free suspension), increased cell growth rate and overall cell yield. The increase in cell yield was associated with a reduced lag phase for all cultures using the cells-on-beads inoculation method, enabling the cells to reach the higher growth phase of the culture quicker in comparison to the control. It was also demonstrated that the cell identity and differentiation potential was retained in all culture medium conditions and with both inoculation methods. Cellson-beads inoculation is clearly an effective means of process intensification and can have a significant impact on reducing overall expansion times and therefore the costs of BM-hMSC and other anchorage-dependent cell therapies. 


\section{Acknowledgements}

This study has been funded by the Engineering and Physical Sciences Research Council via the E-TERM Landscape Fellowship programme (grant no. EP//017801/1) and as part of the Doctoral Training Centre in Regenerative Medicine (grant no. EP/F500491/1), Bioprocessing Research Industry Club (BRIC) (grant No. BB/K011066/1 and BB/K01099/1) and Pall Life Sciences.

\section{Conflict of interest}

The authors declare no financial or commercial conflict of interest. 


\section{Figure captions}

Figure 1: Phase contrast images of the bead-to-bead transfer between the different types of microcarriers, Plastic (white with black rim), Hillex (red) and Cytodex-1 (translucent white). . The white arrows indicate cell-microcarrier aggregates between two different microcarriers. The black arrows indicate single cells attached to a specific microcarrier. Representative images from BMhMSC1 $(A, B, E, F), B M-h M S C 2(C, D, G, H)$ are illustrated. Figures were taken at day 6 after inoculation ( 3 days after adding the second type of microcarrier).

Figure 2: Phase contrast $(A ; C)$ and Live/Dead staining $(B ; D)$ images of the same microcarriers and cells demonstrating the bead-to-bead transfer, Representative images from BM-hMSC1 $(A, B)$ and BM-hMSC2 $(C, D)$ are depicted with similar findings across both donors used. Figures were taken at day 6 after inoculation ( 3 days after adding the second type of microcarrier). The microcarriers used were Plastic (white with black rim) and Hillex (red).

Figure 3: Fluorescent and bright field images showing Cell Tracker Red-labelled cells migrated from Plastic (solid) to Cytodex-1 (transparent) microcarriers (white arrows) and Cell Tracker Green-labelled cells migrated from Cytodex-1 to Plastic microcarriers (yellow arrows). (A) Bright field image; (B) Cell Tracker-Green; (C) Cell Tracker-Red; (D) Merged. Scale bar represents 100 $\mu \mathrm{m}$.

Figure 4: Quantitative demonstration of bead-to-bead culture in FBS and SFM cultures with BMhMSC1 and BM-hMSC2 donor cells cultured on Plastic P102-L microcarriers. Data show mean \pm $\mathrm{SD}, \mathrm{n}=3$.

Figure 5: Cell distribution on microcarriers when using $(A)$ the standard cell inoculation method or (B) the cells-on-beads inoculation method. The gradiation in colour (from light to dark) represents 
the numbers of cells on microcarriers. Data represents BM-hMSC3 cells cultured on Plastic P102$\mathrm{L}$ microcarriers in HPL. The data is representative of findings from the other donor cells and cultured in the other medium compositions.

Figure 6: Comparison of BM-hMSC1 growth on Plastic P102-L microcarriers in a FBS-containing medium when using the standard cell inoculation (control) or cells-on-beads inoculation, where the data represents, (A) the cell density (cells/mL) over 7 days, (B) the mean specific growth rate $\left(d^{-1}\right)$ over the course of the culture and $(C)$ a logarithmic plot of the cell density across the culture period. Data show mean $\pm \mathrm{SD}, \mathrm{n}=3$. Significance was determined at $p<0.05\left(^{*}\right)$ or at $p<0.01$ $(* \star)$.

Figure 7: Comparison of BM-hMSC2 growth on Plastic P102-L microcarriers in the SFM when using the standard cell inoculation (control) or cells-on-beads inoculation, where the data represents, (A) the cell density (cells/mL) over 7 days, (B) the mean specific growth rate $\left(\mathrm{d}^{-1}\right)$ over the course of the culture and $(C)$ a logarithmic plot of the cell density across the culture period. Data show mean $\pm S D, n=3$. Significance was determined at $p<0.05\left(^{*}\right)$ or at $p<0.01\left(^{* *}\right)$.

Figure 8: Comparison of BM-hMSC3 growth on Plastic P102-L microcarriers in a HPL-containing medium when using the standard cell inoculation (control) or cells-on-beads inoculation, where the data represents, (A) the cell density (cells/mL) over 7 days, (B) the mean specific growth rate $\left(d^{-1}\right)$ over the course of the culture and $(C)$ a logarithmic plot of the cell density across the culture period. Data show mean $\pm \mathrm{SD}, \mathrm{n}=3$. Significance was determined at $p<0.05\left(^{*}\right)$ or at $p<0.01$ $(* *)$.

Figure 9: Metabolite data for the cultures comparing the standard cell inoculation (control) and cells-on-beads inoculation across the different culture medium conditions, FBS, HPL and SFM.

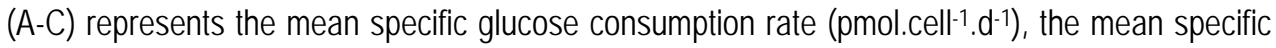

Formatted: Font color:

Formatted: Font color:

Auto

Formatted: Font color:

Auto

Formatted: Font color:

Formatted: Font color:

Auto

Formatted: Font color:

Auto

Formatted: Font color:

Auto 
lactate production rate (pmol.cell-1. $\mathrm{d}^{-1}$ ) and the mean specific ammonia production rate (pmol.cell${ }^{1} . \mathrm{d}^{-1}$ ) for the FBS culture. (D-F) represents the mean specific glucose consumption rate (pmol.cell$\left.{ }^{1} \cdot \mathrm{d}^{-1}\right)$, the mean specific lactate production rate $\left(\right.$ pmol.cell-1. $\left.\mathrm{d}^{-1}\right)$ and the mean specific ammonia production rate (pmol.cell-1. $\mathrm{mL}^{-1}$ ) for the HPL culture. (G-I) represents the mean specific glucose consumption rate $\left(\mathrm{pmol} . \mathrm{cell}^{-1} \cdot \mathrm{mL}^{-1}\right)$, the mean specific lactate production rate $\left(\mathrm{pmol} . \mathrm{cell}^{-1} \cdot \mathrm{d}^{-1}\right)$ and the mean specific ammonia production rate $\left(\mathrm{pmol}^{\mathrm{c}} \mathrm{ell}^{-1} \mathrm{~d}^{\mathrm{d}}\right.$ ) for the SFM culture. Data show mean $\pm S D, n=3$.

Supplementary Figure 1: Immunophenotypic expression for BM-hMSC1 (A) and differentiation of the BM-hMSC1 toward the (B) adipogenic, (C) osteogenic and (D) chondrogenic lineages. All data are of cells cultured on Plastic P102-L microcarriers. Representative data was obtained for all other cell donors and in all other medium conditions.

Formatted: Font color:

Auto

Formatted: Font color:

Formatted: Font color:

Formatted: Font color:

Formatted: Font color:

Auto 


\section{References}

Badenes SM, Fernandes TG, Miranda CC, Pusch-Klein A, Haupt S, Rodrigues CAV, Diogo MM, Brüstle O, Cabral JMS. 2017. Long-term expansion of human induced pluripotent stem cells in a microcarrier-based dynamic system. Journal of Chemical Technology \& Biotechnology 92(3):492-503.

Bardy J, Chen AK, Lim YM, Wu S, Wei S, Weiping H, Chan K, Reuveny S, Oh SK. 2013. Microcarrier suspension cultures for high-density expansion and differentiation of human pluripotent stem cells to neural progenitor cells. Tissue Eng Part C Methods 19(2):166-80.

Barry F, Murphy M. 2013. Mesenchymal stem cells in joint disease and repair. Nat Rev Rheumatol 9(10):584-594.

Chan AK, Heathman TR, Coopman K, Hewitt CJ. 2014. Multiparameter flow cytometry for the characterisation of extracellular markers on human mesenchymal stem cells. Biotechnol Lett 36(4):731-41.

Chen X, Chen A, Woo TL, Choo AB, Reuveny S, Oh SK. 2010. Investigations into the metabolism of two-dimensional colony and suspended microcarrier cultures of human embryonic stem cells in serum-free media. Stem Cells Dev 19(11):1781-92.

de Soure AM, Fernandes-Platzgummer A, da Silva CL, Cabral JMS. 2016. Scalable microcarrierbased manufacturing of mesenchymal stem/stromal cells. Journal of Biotechnology 236:88-109.

Dominici M, Le Blanc K, Mueller I, Slaper-Cortenbach I, Marini F, Krause D, Deans R, Keating A, Prockop D, Horwitz E. 2006. Minimal criteria for defining multipotent mesenchymal stromal cells. The International Society for Cellular Therapy position statement. Cytotherapy 8(4):315-7.

Dos Santos F, Andrade PZ, Abecasis MM, Gimble JM, Chase LG, Campbell AM, Boucher S, Vemuri MC, Silva CL, Cabral JM. 2011. Toward a clinical-grade expansion of mesenchymal stem cells from human sources: a microcarrier-based culture system under xeno-free conditions. Tissue Eng Part C Methods 17(12):1201-10.

Heathman TRJ, Glyn VAM, Picken A, Rafiq QA, Coopman K, Nienow AW, Kara B, Hewitt CJ. 2015. Expansion, harvest and cryopreservation of human mesenchymal stem cells in a serum-free microcarrier process. Biotechnology and Bioengineering 112(8):1696-1707.

Heng BC, Cowan CM, Basu S. 2009. Comparison of enzymatic and non-enzymatic means of dissociating adherent monolayers of mesenchymal stem cells. Biol Proced Online 11:161-9.

Hervy M, Weber JL, Pecheul M, Dolley-Sonneville P, Henry D, Zhou Y, Melkoumian Z. 2014. Long Term Expansion of Bone Marrow-Derived hMSCs on Novel Synthetic Microcarriers in Xeno-Free, Defined Conditions. PLoS ONE 9(3):e92120.

Huang $\mathrm{H}-\mathrm{L}$, Hsing H-W, Lai T-C, Chen Y-W, Lee T-R, Chan H-T, Lyu P-C, Wu C-L, Lu Y-C, Lin S-T and others. 2010. Trypsin-induced proteome alteration during cell subculture in mammalian cells. Journal of Biomedical Science 17(1):36-36.

Kapiszewska M, Reddy NM, Lange CS. 1991. Trypsin-induced changes in cell shape and chromatin structure result in radiosensitization of monolayer Chinese hamster V79 cells. Int J Radiat Biol 60(4):635-46.

Leber J, Barekzai J, Blumenstock M, Pospisil B, Salzig D, Czermak P. 2017. Microcarrier choice and bead-to-bead transfer for human mesenchymal stem cells in serum-containing and chemically defined media. Process Biochemistry 59:255-265.

Lindner E, Arvidsson AC, Wergeland I, Billig D. 1987. Subpassaging cells on microcarriers: the importance for scaling up to production. Dev Biol Stand 66:299-305. 
Madeira A, Da Silva CL, Dos Santos F, Camafeita E, Cabral JM, Sa-Correia I. 2012. Human mesenchymal stem cell expression program upon extended ex-vivo cultivation, as revealed by 2-DE-based quantitative proteomics. PLoS One 7(8):e43523.

Nienow AW, Rafiq QA, Coopman K, Hewitt CJ. 2014. A potentially scalable method for the harvesting of hMSCs from microcarriers. Biochemical Engineering Journal 85(0):79-88.

Ohlson S, Branscomb J, Nilsson K. 1994. Bead-to-bead transfer of Chinese hamster ovary cells using macroporous microcarriers. Cytotechnology 14(1):67-80.

Rafiq QA. 2016. Toward a scalable and consistent manufacturing process for the production of human MSCs. Cell Gene Therapy Insights 2(1):127-140.

Rafiq QA, Brosnan KM, Coopman K, Nienow AW, Hewitt CJ. 2013a. Culture of human mesenchymal stem cells on microcarriers in a 5 I stirred-tank bioreactor. Biotechnol Lett 35(8):1233-1245.

Rafiq QA, Coopman K, Nienow AW, Hewitt CJ. 2013b. A quantitative approach for understanding small-scale human mesenchymal stem cell culture - implications for large-scale bioprocess development. Biotechnol J 8(4):459-71.

Rafiq QA, Coopman K, Nienow AW, Hewitt CJ. 2016. Systematic microcarrier screening and agitated culture conditions improves human mesenchymal stem cell yield in bioreactors. Biotechnology Journal 11(4):473-486.

Takahashi I, Sato K, Mera H, Wakitani S, Takagi M. 2017. Effects of agitation rate on aggregation during beads-to-beads subcultivation of microcarrier culture of human mesenchymal stem cells. Cytotechnology 69(3):503-509.

Wang Y, Ouyang F. 1999. Bead-to-bead transfer of Vero cells in microcarrier culture. Cytotechnology 31(3):221-224.

Yamakita Y, Totsukawa G, Yamashiro S, Fry D, Zhang X, Hanks SK, Matsumura F. 1999. Dissociation of FAK/p130(CAS)/c-Src Complex during Mitosis: Role of Mitosis-specific Serine Phosphorylation of FAK. The Journal of Cell Biology 144(2):315-324. 